\title{
Spiders (Aranei) of the Arctic Island Sibiryakova, Kara Sea, Russia
}

\section{Пауки арктического острова Сибирякова, Карское море, Россия}

\author{
Andrei V. Tanasevitch ${ }^{1}$, Leonid B. Rybalov ${ }^{1}$, Iraida G. Vorobeva ${ }^{2}$ \\ А.В. Танасевич ${ }^{1}$, А.Б. Рыбалов ${ }^{1}$, И.Г. Воробьева ${ }^{2}$
}

\author{
${ }^{1}$ A.N. Severtsov Institute of Ecology and Evolution, Russian Academy of Sciences, Leninsky prospekt 33, Moscow 119071, Russia. E- \\ mails: tanasevitch@gmail.com; lrybalov52@mail.ru \\ Институт проблем экологии и эволюции имени А.Н. Северцова РАН, Ленинский проспект 33, Москва 119071, Россия. \\ ${ }^{2}$ Mari State University, Yoshkar-Ola, Lenina Sq. 1. vigir@mail.ru \\ Марийский государственный университет, Йошкар-Ола, пл. Ленина 1.
}

KEY WORDS: Russian Arctic, tundra, chorology, check-list, pitfall trapping, soil sampling.

КЛЮЧЕВЫЕ СЛОВА: Российская Арктика, тундра, хорология, список видов, ловушки Барбера, почвенные пробы.

ABSTRACT. The Sibiryakova Island was found to be inhabited by 13 spider species of a single family Linyphiidae. In the composition of zoogeographic elements, the island fauna can be characterized as Siberian and consisting mostly of species with Siberian and Siberian-Nearctic ranges. In the composition of zonal fractions, the fauna is arctic and represented mainly by pure arctic components (11 species), with a small proportion of polyzonal species (2). Hypoarctic and boreal elements are absent. The study concerned the fauna and inhabitants of seven different types of vegetative communities: zonal (3) and intrazonal (4). The zonal (most pessimal) community - the moss-lichen polygonal tundra - is characterized, as compared to other areas, by the least number of species found (4) and by the least abundance of their populations (the exception is Halorates spetsbergensis (Thorell, 1872)). The vast majority of the fauna (10 species) and population is concentrated in the most wet intrazonal biotopes - the moss-cotton grass polygonal tundra. Three species, Erigone psychrophila Thorell, 1872, H. spetsbergensis and Mecynargus tundricola Eskov, 1988, explore the whole range of biotopes, both zonal and intrazonal, though the highest dynamic density/activity is seen only in the latter ones. It was confirmed that the spider fauna in wet intrazonal biotopes is considerably richer and their inhabitants are more abundant and represented by a wider range of latitudinal fractions than in other kinds of vegetative communities of the arctic tundras. The dynamic density/activity of spiders is more than twice in intrazonal communities as compared to zonal biotopes. The pitfall traps are responsible for all the 13 island spider species found, the soil samples (40) found only 7 species. The density of spider population comprised from 10 specimens/sq. m. in swampy mosslichen tundra to 46 specimens/sq. m. in moss-lichen polygonal one. Spiders are the dominating group of the soil macrofauna of arctic tundras. Based on the results of the pitfall trap catching, their proportion in the col- lection of land invertebrates comprised $60-91 \%$ in early July and $10-49 \%$ in the second part of July. Spiders comprise $27-63 \%$ of the macrofauna in the soil samples. Each of the 13 spider species of the Sibiryakova Island is characterized by distribution between the vegetation types, the range and belonging to the zonal fraction are indicated.

In addition, the species Agyneta decora (O. Pickard-Cambridge, 1871) is recorded in the Yamal Peninsula (Sabetta, northern Yamal) for the first time.

How to cite this article: Tanasevitch A.V., Rybalov L.B., Vorobeva I.G. 2020. Spiders (Aranei) of the Arctic Island Sibiryakova, Kara Sea, Russia // Arthropoda Selecta. Vol.29. No.1. P.141-150. doi: 10.15298/arthsel. 29.1.13

РЕЗЮМЕ. На острове Сибирякова найдено 13 видов пауков одного семейства Linyphiidae. По составу зоогеографических элементов фауну острова можно охарактеризовать как сибирскую, составленную в большинстве своём видами с сибирскими и сибирско-неарктическими ареалами. По составу зональных фракций фауна арктическая, представлена в основном чисто арктическими элементами (11 видов), с малым участием полизональных форм (2 вида). Гипоарктические и бореальные элементы отсутствуют. Изучена фауна и население семи различных типов растительных сообществ: зональных (3) и интразональных (4). Зональное (наиболее пессимальное) сообщество - мохово-лишайниковая полигональная тундра, — по сравнению с остальными участками, характеризуется наименьшим числом найденных здесь видов (4) и наименьшей численностью их популяций (исключение Halorates spetsbergensis (Thorell, 1872)). Бо́льшая часть фауны (10 видов) и населения сосредоточена в наиболее влажном интразональном биотопе - моховопушицевой полигональной тундре. Три вида, Erigone psychrophila Thorell, 1872, H. spetsbergensis и 


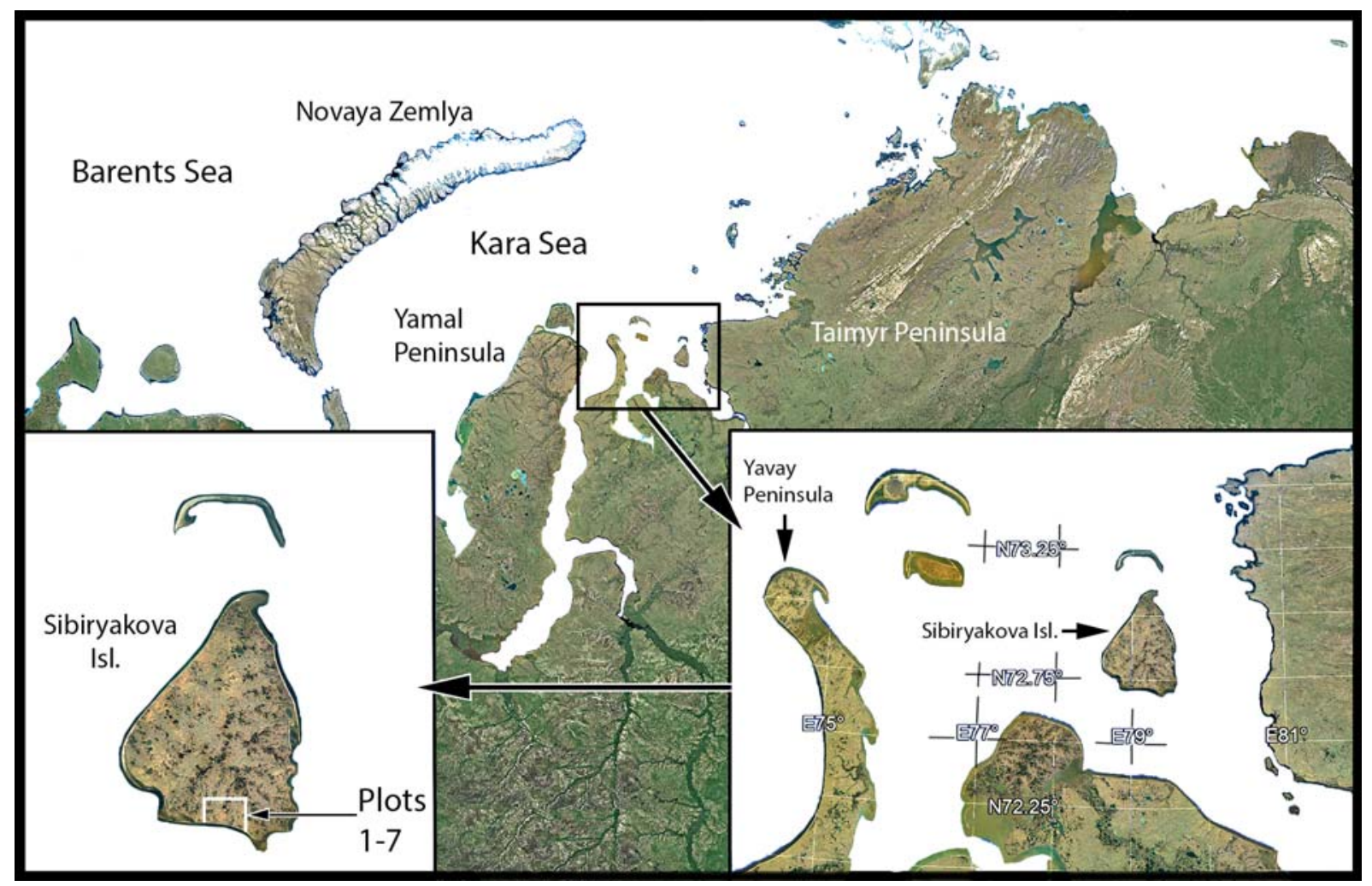

Map. Collecting plots on the Sibiryakova Island.

Карта. Месторасположение площадок на о-ве Сибирякова.

Mecynargus tundricola Eskov, 1988 осваивают весь спектр биотопов, как зональных, так и интразональных, но наибольшую динамическую плотность показывают лишь в последних. Подтверждено, что во влажных интразональных биотопах фауна пауков существенно богаче, их население обильнее и представлено более широким спектром широтных фракций, чем в других типах растительных сообществ арктических тундр. Динамическая плотность пауков в интразональных сообществах более чем вдвое превышает этот показатель в зональных биотопах. Ловушками Барбера собраны все 13 видов пауков острова, почвенными пробами (40 проб) учтено лишь 7. Плотность населения пауков составила от 10 экз./кв.м. в заболоченной мохово-лишайниковой тундре до 46 экз./кв.м. в мохово-лишайниковой полигональной. Пауки - доминирующая группа почвенной мезофауны местных арктических тундр. По данным сбора ловушками на их долю в начале июля приходится 60-91\% всех пойманных наземных беспозвоночных, во второй половине июля - 10-49\%. В почвенных пробах пауки составляют 27-63\% всей мезофауны. Для каждого из 13 видов пауков о-ва Сибирякова приведено распределение по типам растительности, указан тип ареала и принадлежность к зональной фракции.

В дополнение, вид Agyneta decora (O. PickardCambridge, 1871) впервые указан для п-ова Ямал (Сабетта, северный Ямал).

\section{Introduction}

Among islands of Russian Arctic beyond the archipelagos, there are perhaps only four relatively well studied in respect to araneofauna: Bolshoy Aynov and Dolgiy islands in the Barents Sea; Vaygach and Shokalsky islands in Kara Sea, and Wrangel Island in the Chukchi Sea. The fauna of spiders of Bolshoy Aynov comprises 17 species [Nekhaeva, Nekhaev, 2011], that of Dolgiy - 52 [Marusik et al., 2016], Vaygach — 21 [Tanasevitch, 2017b], Shokalsky - 14 [Nekhaeva, 2018] and Wrangel - 37 species [Khruleva, 1987]. Besides the species lists, the authors revealed some zoogeographic peculiarities of the fauna, specified the ranges of its representatives, detected some chorological groups and their distribution in zonal-landscape types of vegetation. Other similar islands of Russian Arctic (beyond the archipelagos) are very unsatisfactorily studied: Kolguev Island is known as inhabited by only one species [Tanasevitch, 2017a], Oleniy — by 3 [Tanasevitch, 2017a], and Belyi — by five species [Holm, 1970; Tanasevitch, 2017a].

Among islands of Russian Arctic within archipelagos, Novaya Zemlya can be regarded the most studied: 23 species [Tanasevitch, 2017b, 2018]. Inventories of other archipelagos are considerably shorter: Franz Josef Land — two species [Bulavintsev, Babenko, 1983], Severnaya Zemlya - three [Eskov, 1985], and Novosibirskiye Ostrova - five [Eskov, 1985; Marusik et 
al., 1993]. At the same time it cannot be expected that the spider fauna of these archipelagos will considerably increase because they are situated in the polar deserts zone or in the arctic tundra subzone [Matveyeva, 1998], which are characterized by extremal existence conditions and, respectively, a very poor biota.

\section{Material, methods and sampling}

The present paper is based on the extensive material collected by I. Vorobeva on the Sibiryakova Island (see the Map) in the period from June 29 to August 1, 1989. Spiders were collected by soil sampling, pitfall trapping and by hand collecting. Pitfall traps $(200 \mathrm{ml}$ plastic cups, filled at $2 / 3$ by $2 \%$ formaldehyde solution) were positioned in seven studied biotopes in lines of 5-10 traps. In total, 2536 adult spider specimens were gained and examined. The data on lines with 5 and 10 traps were reconsidered for 100 trap/days. The text contains data on adult (identified) spiders, nonadult specimens were accounted in the total number (soil samples) or in the dynamic density (pitfall traps). The macrofaunal soil samples were taken at the area of $0.25 \times 0.25 \mathrm{~m}$, layer-by layer, 8 samples per plot. In total, 40 samples were taken on plots No. 2 to No. 7 .

Range types of spiders are given according to Tanasevitch \& Rybalov [2015]; Tanasevitch \& Nekhaeva [2016] and Tanasevitch \& Khruleva [2017] with a few additions and corrections.

Abbreviations used in the text: PCD - projective cover of vegetation degree; TPCD - total projective cover of vegetation degree.

\section{Region and localities}

The Sibiryakova Island is situated in the southern Kara Sea at the exit from the Yenisei River Bay, between Gydan and Taimyr peninsulas. This is an alluvial plain island with a flat relief and a number of thermokarst lakes and small rivers. Their by-mouth areas are represented by saline short-grass meadows littered by a huge amount of driftwoods taken out from the Yenisei River; the plains covered by arctic tundras. Micro- and nanorelief permafrost. There is no frostfree period. The soils are subsoil-frozen tundra-sandy, subsoil-frozen tundra-boggy and oozy, always weak in thickness, often with dense root mat. The vegetation is rather variable: 15 species of mixomicetes, 71 species of lichens, 83 species of mosses, 160 species and subspecies of vesicular plants [Kuvaev et al., 1994; Matveyeva, Zanokha, 1984].

According to geobotanical regionalization, the Sibiryakova Island lies in the arctic tundra subzone of the tundra zone [Matveyeva, 1998].

NOTE: 1989 summer was extremely cold even for the Arctic [Kuvaev et al., 1994], which probably decreased the probability of revealing the more number of spider species, as well as their activity and numbers.

\section{Typification of plant communities}

The scheme proposed by Yuri I. Chernov [1978] for classifying plant communities of the Arctic has been used for the analysis of zonal-landscape distribution of tundra spiders. Based on this classification, there are three main zonal-landscape types of plant communities in the tundra: zonal, intrazonal and azonal (for more details, see Tanasevitch \& Koponen [2007]; Tanasevitch \& Rybalov [2015]; Tanasevitch \& Khruleva [2017]). Brief description of the types of vegetation on the Sibiryakova Island is given below.

Zonal plant communities (Zonal in text below) the plant communities that are restricted to the watershed of one zone (subzone), defining the vegetation zonal face of the landscape. In zonal communities, abiotic factors are more extreme as compared to those in intrazonal communities and therefore living conditions are more adverse. In the investigated area, the zonal plant associations are represented by different variants of undershrub-sedge-lichen-moss tundra rare with spots of bare ground.

In the studied area, the intrazonal communities are represented by moss-lichen-, dwarf willow-moss-sedgeand undershrub-moss-lichen polygonal tundra on watershed.

The numbers of studied plots are given in the order of their increasing humidity.

Plot No. 1. Moss-lichen polygonal tundra on watershed with spots of bare ground (sand) on left bank of Lagernaya River, $72.722819^{\circ} \mathrm{N} 79.258800^{\circ} \mathrm{E}$, [1]. Polygons ca. 6-10 $\mathrm{m}$ in diameter, bordered with swampy hollows. Spots of bare sand ca. 5-10\% of biotope. Total projective cover degree (TPCD ) ca. 95\%. Tundra communities with domination of lichens, projective cover degree (PCD) ca. 75-80\%: Parmelia stygia, Alectoria nigricans, Pertusaria sp., Thamnolia vermicularis, etc.; mosses PCD ca. 10\%: Polytrichum strictum, Hylocomium splendens var. alascanum, Dicranum spadiceum, etc. Angiospermae ca. PCD 10-15\%): Novosieversia glacialis, Dryas punctata, Salix nummularia, Lloydia serotina, Luzula confuse, Potentilla hypartica, Saxifraga foliolosa, Hieróchloë alpina. Soil: gleyic podburs (enti-gleyic podzols); soil texture sand. This site first freed from the snow and thawed.

Plot No. 2. Undershrub-moss-lichen polygonal tundra on left bank of Lagernaya River, $72.713939^{\circ} \mathrm{N}$ $79.312019^{\circ} \mathrm{E},[2]$. TPCD ca. $100 \%$. Tundra communities with domination of lichens (PCD ca. 60\%): $\mathrm{Ce}$ traria cucullata, Cetraria tenuiàolia, Cetraria islandica, Cladonia amaurocraea, Cladonia mitis,Cladonia gracilis, Dactylina arctica, Alectoria nigicans, etc.; mosses (PCD ca. 20\%): Dicranum spadiceum, Aulacomnium turgidum, Polytrichum alpinum, Hylocomium splendens var. alascanum, etc.; dwarf shrubs and grasses (PCD ca. 20\%): Salix nummularia (PCD ca. 10\%), Dryas punctata (PCD ca. 6\%), Vaccinium vitisidea (PCD ca. 1\%), Polygonum viviparum (PCD ca. 3\%), Luzula confusa (PCD ca. 3\%), L. wahlenbergii, etc. Soil: peat gleyzems (histic gleysols); soil texture 
- sand, excessively moistened. Permafrost at the middle summer ca. $12-15 \mathrm{~cm}$.

Plot No. 3. Dwarf willow-moss-sedge polygonal tundra on gentle S-slope $\left(1-2^{\circ}\right)$ of watershed, 15-20 m a.s.1, $72.724371^{\circ} \mathrm{N} 79.167811^{\circ} \mathrm{E}$, [3]. Polygons ca. $10-15 \mathrm{~m}$ in diameter. There are small hollows with water. TPCD ca. $100 \%$, Salix pulchra (40\%), S. repens (PCD ca. 30\%), Luzula confusa (PCD ca. 10\%), L. wahlenbergii (PCD ca. 10\%), Pedicularis sudetica (PCD ca. 1-2\%), Saxifraga nivalis (PCD ca. 1\%); mosses (PCD ca. 3\%): Polytrichum strictum, Aulacomnium turgidum, Hylocomium splendens var. alascanum; lichens (PCD ca. 1\%): Dactylina arctica, Pertusaria sp., Nephroma arcticum. Soil: gleyic podburs (enti-gleyic podzols); soil texture - sand, well drained.

Intrazonal plant communities (Intra in text below) — the plant communities that are distributed across one or several zones (subzones); the typical examples being swamps and river valley vegetation: e.g., willow stands in flood-plains, meadows, rocky and/or grassy slopes, forest stands on river banks or river terraces, etc. Commonly, parts of intrazonal communities are situated on the watershed among zonal vegetation types: these are willow shrubs in depressions, shrubby lake shores, flat-hill peatbogs with sedge fens, or sphagnum bogs in depressions, grassy slopes of small brooks, etc. The intrazonal communities are characterized by a smoother gradient of climatic factors (especially, of temperature and humidity), making living conditions there significantly less pessimal.

In the studied area, the intrazonal communities are represented by grass-undershrub- and undershrub-grassmoss-lichen tundra on bank terraces, as well as by swampy moss-lichen tundra on watershed.

Plot No. 4. Grass-dwarf shrubs tundra on first Sslope $\left(5-7^{\circ}\right)$ terrace of right bank of Lagernaya River, $72.732500^{\circ} \mathrm{N} 79.245069^{\circ} \mathrm{E},[4]$, with bare sandy spots occupy $50 \%$ of biotope. TPCD ca. $50 \%$ : grass associations with dwarf shrubs: Dryas punctata (PCD ca. 10\%), Salix nummularia (PCD ca. 10\%), Minuartia arctica (PCD ca. 1\%), Novosieversia glacialis (PCD ca. 5\%), Luzula confusa (PCD less than 1\%), Polygonum viviparum (PCD less than 1\%), Arctargostis latifolia (PCD less than 1\%), Deschampsia glauca (PCD less than less 1\%), Alopecurus alpinus (PCD ca. 1\%); sporadic: Trisetum spicatum, Androsace bungeana, Artemisia tilesii, Lloydia serotina; sporadic: mosses \& lichens. Soil: podburs (enti-umbcic podzols); soil texture sand.

Plot No. 5. Dwarf shrubs-grass-moss-lichen tundra on gentle slope of bank in valley of Izvilistaya River $\left(72.759689^{\circ} \mathrm{N} 79.176889^{\circ} \mathrm{E}\right)$, [5]. Microrelief well-developed hummocks/pingos, poligons of loam ca. 15$20 \mathrm{~m}$, occupy $40 \%$ of biocenosis. TPCD ca. 60\%: lichens (PCD ca. 40-50\%): Alectoria nigricans, Pertusaria sp., Parmelia stypia, Dactylina arctica, Cetraria nivalis, Ochrolechia frigida; undershrubs \& grasses (PCD ca. 10-20\%): Salix nummularia (PCD ca. 15\%), Luzula confusa (PCD ca. 1-2\%), Ryas punctata (PCD ca. 1-2\%), Oxyria digyna (PCD less than
1\%), Festuca cryophila (PCD less than 1\%), Draba nivalis \& Polygonum viviparum (PCD ca. 2\%), Potentilla hyparctica (PCD ca. less than 1\%); sporadic: Pedicularis oederi, Pedicularis hirsute, Poa alpigena, Saxifraga cernua, Lloydia serotina, Minuartia arctica; mosses: Dicranum spadiceum, Polytrichum strictum. Soil: cryozems (calcic cryosols); soil texture - sand.

Plot No. 6. Wet moss-cotton-grass polygonal tundra on edge of watershed in middle flow of Izvilistaya River, $72.744419^{\circ} \mathrm{N} 79.135550^{\circ} \mathrm{E}$, at distance of $60 \mathrm{~m}$ from bay bank, [6]. There are small hollows with water. TPCD ca. 100\%: Eriophorum angustifolium \& E. scheuchzeri (PCD ca. 95\%), Calamagrostis holmii (PCD ca. 2-3\%), Polygonum viviparum (PCD ca. 1\%), Carex ensifolia (PCD ca. 1\%), sporadic: Poa arctica, Saxifraga foliolosa, Pedicularis hirsute, Lycopodium selago; mosses: Dicranum spadiceum, Drepanocladus uncinatus, Aulacomnium turgidum; lichens: Cetraria sp., Dactylina arctica, Nephroma arcticum. Soil: peat gleyzems (histic gleysols), excessively moistened.

Plot No. 7. Swampy moss-lichen tundra in shallow depression on watershed, $72.744731^{\circ} \mathrm{N} 79.190139^{\circ} \mathrm{E}$, [7]. Swampy spots ca. 15-20 m, separated with sedgeEriophorum communities ca. 1.5-2 m wide. TPCD ca. 100\%. Mosses and lichens (PCD ca. 95\%): Dicranum spadicium, Aulacomnium turgidum, Sphagnum sp., Polytrichum strictum, Drepanocladus uncinatus; Cladonia gracilis, C. amaurocraea, C. rangiferina, Cetraria delisei, $C$. islandica var. arctica, Thamnolia vermicularis. Angiospermae sporadic (PCD ca. 5\%): Salix polaris (PCD ca. 1-2\%), Luzula wahlenbergii (PCD ca. 1\%), Saxifraga nivalis (PCD ca. 1\%). Polygonum viviparum (PCD ca. 1\%). Soil: peat gleyic soil (gleyi-histic albeluvisols), with thick layer of peat ca. 15-18 cm; soil texture - loamy sand.

NOTE: The data on population in plot No. 7 are not included in the tables of calculation of the numbers an in the general analysis of the fauna and population because this area of tundra was flooded for nearly the entire summer of 1989, which has led to impossibility of soil sampling and exposing the pitfall traps during the main period of investigations. Therefore, the data on the plot No. 7 are only informative.

Azonal communities - the plant communities that are not confined by a certain zone (subzone) but distributed across many zones (subzones), and usually represented by specific beach plant communities on seashore, marshlands, and pebble banks of rivers and seas. This type of communities has not been addressed in this investigation.

\section{List of spiders of the Sibiryakova Island}

Families are given in the alphabetic order. Abbreviations Zonal and Intra correspond to investigated types of plant communities, numbers in square brackets correspond to numbers of certain above-described biotopes. Numbers following after "pitfall traps" mean a number of traps in line. 


\section{Agyneta decora (O. Pickard-Cambridge, 1871)}

MATERIAL. Intra. $2 \bigcirc^{7} \sigma^{7}$, grass-dwarf shrubs tundra on terrace [4], pitfall traps 5, 2-11.VII.1989.

REMARKS. Known from the southern tundra subzone on Dolgiy Island [Marusik et al., 2016] and Taymyr [Osipov, 2003], as well as from the arctic tundra subzone in Yakutia [Tanasevitch, Nekhaeva, 2016], and Yamal Peninsula: $2 \sigma^{7} \sigma^{7}$, northern Yamal, environs of Sabetta, 71. $237528^{\circ} \mathrm{N} 72.113524^{\circ} \mathrm{E}$, slightly disturbed sphagnum-cloudberry (Sphagnum sp. and Rubus chamaemorus) plant community, pitfall traps, 16-27.VII.2017, leg. L. Rybalov and A. Bastrakov (new locality!). In the Nearctic, A. decora was recorded from Alaska and Yukon Territory [Dupérré, 2013]. The species is new for the fauna of the Yamal Peninsula.

RANGE. Palaearctic-West Nearctic polyzonal.

\section{Arcterigone pilifrons (L. Koch, 1879)}

MATERIAL. Zonal. $11 O^{7} O^{7}, 4$ 우, dwarf willow-moss-sedge polygonal [3], pitfall traps 5, 29.VI.-8.VII.1989; $1 \sigma^{x}, 1$ ㅇ, [3] pitfall traps 5, 21-31.VII.1989; $14 \mathrm{O}^{\top} \mathrm{O}^{\top}, 4$ 우, [3], pitfall traps 10, 29.VI.-8.VII.1989; $1 \mathrm{O}^{7}, 1$ ㅇ, [3], soil sample No. 21, layer 0-1 cm, 10.VII.1989; $1 \mathrm{O}^{7}$, [3], soil sample No. 23, layer 1-4 cm, 10 . VII.1989.

Intra. $1 \bigcirc^{\top}, 1+$, wet moss-cotton-grass polygonal tundra [6], pitfall traps 10, 29.VI.-8.VII.1989; $3 \sigma^{7} \sigma^{7}, 2$ OO , [6], pitfall traps 5, 29.VI.-8.VII.1989.

RANGE. Siberian-West Nearctic arctic.

\section{Diplocephalus barbiger (Roewer, 1955)}

MATERIAL. Zonal. $1 \sigma^{7}$, undershrub-moss-lichen polygonal tundra [2], pitfall traps 10, 20-30.VII.1989.

Intra. $7 O^{7} \sigma^{7}, 2$, 2 , dwarf willow-moss-sedge polygonal tundra [4], pitfall traps 5, 29.VI.-8.VII.1989; $1 \sigma^{7}, 1$ \%, grass-dwarf shrubs tundra on terrace [5], pitfall traps 5, 2-11.VII.1989; 5 O $^{7} \sigma^{7}$ 1 , [5], pitfall traps 10, 2-11.VII.1989; 1 ㅇ, [5], pitfall traps 5, 21-31.VII.1989; 2 O$^{7} \sigma^{7}, 4$ OP, [5], pitfall traps 10, 21-31.VII.1989; $29 \mathrm{O}^{7} \mathrm{O}^{7}$, wet moss-cotton-grass polygonal tundra [6], pitfall traps 5, 29.VI.-8.VII.1989; 80 O' $^{7}, 9$ OP, [6], pitfall traps 10, 29.VI.8.VII.1989; 2 +क , [6], pitfall traps 5, 21-31.VII.1989; $6 \mathrm{O}^{7} \bigcirc^{7}$, [6], pitfall traps 10, 20-31.VII.1989.

RANGE. Siberian-Nearctic arctic.

\section{Erigone arctica palaearctica Braendegaard, 1934}

MATERIAL. Zonal. $8 \sigma^{7} \sigma^{7}$, dwarf willow-moss-sedge polygonal tundra [3], pitfall traps 5, 29.VI.-8.VII.1989; 3 +क, [3], pitfall traps 5, 21-31.VII.1989; $1 \sigma^{7}, 1 \%$, [3], pitfall traps 5, 21-31. VII.1989; 3 우, [3], pitfall traps 5, 21-31.VII.1989; 4 우, [3], pitfall traps 10, 29.VI.-8.VII.1989.

Intra. $30^{7} \sigma^{7}, 10$ 우, grass-dwarf shrubs tundra on terrace [4], pitfall traps 5, 2-11.VII.1989; 1 \% , [4], pitfall traps 5, 21-31. VII.1989; $10^{0^{7},}, 4$ 우, [4], pitfall traps 10, 21-31.VII.1989; $15 \mathrm{O}^{7} \mathrm{O}^{7}$, 25 우, [4], pitfall traps 10, 21-31.VII.1989; 1 ㅇ, swampy mosslichen tundra in depression on watershed [7], soil sample No. 32, layer 0-1 cm, 26.VII.1989.

RANGE. East European arctic.

\section{Erigone psychrophila Thorell, 1872}

MATERIAL. Zonal. $1+$, moss-lichen polygonal tundra [1], pitfall traps 5, 20-30.VII.1989; $1 \sigma^{7}$, [1], pitfall traps 10, 2030.VII.1989; 2 +, undershrub-moss-lichen polygonal tundra [2] pitfall traps 5, 29.VI.-8.VII.1989; $2 \sigma^{7} \sigma^{7}, 4$ 우, [2], pitfall traps 5, 20-30.VII.1989; $2 \mathrm{O}^{7} \mathrm{O}^{7}, 4$ 우, [2], pitfall traps 10, 29.VI--8. VII.1989; $24 \mathrm{O}^{7} \mathrm{O}^{\top}, 15$ 우, dwarf willow-moss-sedge polygonal tundra [3], pitfall traps 5, 29.VI.-8.VII.1989; $90^{7} \sigma^{7}, 11$ 우, [3], pitfall traps 5, 21-31.VII.1989; $44 \mathrm{O}^{\top} \sigma^{\top}, 26$ 우, [3], pitfall traps 10, 29.VI.-8.VII.1989.

Intra. $15 \sigma^{7} \sigma^{7}, 24 \circ 9$, grass-dwarf shrub tundra on terrace [4], pitfall traps 10, 21-31.VII.1989; $3 \mathrm{O}^{7} \mathrm{O}^{7}, 4$ +9 , dwarf shrubs-grassmoss-lichen tundra [5], pitfall traps 5, 2-12.VII.1989; 14 O $^{7} 0^{7}, 3$ 우, [5], pitfall traps 10, 2-11.VII.1989; $1 \bigcirc^{7}, 1$, [5], pitfall traps 10, 21-31.VII.1989; $26 \bigcirc^{7} \sigma^{7}, 6$ 우, wet moss-cotton-grass polygonal tundra [6], pitfall traps 5, 29.VI.-8.VII.1989; $21 \sigma^{7} \sigma^{7}, 25$ 우, [6], pitfall traps 5, 21-31.VII.1989; $40 \sigma^{7} \sigma^{7}, 25$ 우, [6], pitfall traps 10, 29.VI.-8.VII.1989; $7 \sigma^{\top} \sigma^{7}, 4$ 우, swampy moss-lichen tundra in depression on watershed [7], pitfall traps 10, 22.VII.-1. VIII.1989.

RANGE. Holarctic arctic.

\section{Gibothorax tchernovi Eskov, 1989}

MATERIAL. Intra. $17 \bigcirc^{7} \sigma^{7}, 18$ 우, wet moss-cotton-grass polygonal tundra [6], pitfall traps 5, 21-31.VII.1989; 5 우, [6], pitfall traps 10, 29.VI.-8.VII.1989; $22 \bigcirc^{7} \bigcirc^{7}, 35$ क+ , [6], pitfall traps 10, 20-31.VII.1989.

RANGE. Siberian arctic.

\section{Halorates spetsbergensis (Thorell, 1872)}

MATERIAL. Zonal. $5 \sigma^{7} \sigma^{7}, 90$ 우, moss-lichen polygonal tundra [1], pitfall traps 5, 29.VI.-8.VII.1989; 5 ○ $^{7} \sigma^{7}, 40$ 우, [1], pitfall traps 5, 20-30.VII.1989; 12 O $^{\top} \sigma^{7}, 60$ 우, [1], pitfall traps 10, 29.VI.-8.VII.1989; 7 O $^{7} \sigma^{7}, 60$ 우, [1], pitfall traps 10, 2030.VII.1989; $2 \sigma^{7} \sigma^{7}$, undershrub-moss-lichen polygonal tundra [2], pitfall traps 5, 29.VI.-8.VII.1989; 3 OQ , [2], pitfall traps 5, 2030.VII.1989; 12 O $^{\top} \sigma^{\top}, 18$ OP, [2], pitfall traps 10, 29.VI.-8.VII.1989; 5 우, [2], pitfall traps 10, 20-30.VII.1989; $60^{7} 0^{7}, 9$ 우, dwarf willow-moss-sedge polygonal tundra [3], pitfall traps 5, 29.VI.8.VII.1989; 1 O$^{7}, 1$ \&, [3], pitfall traps 5, 21-31.VII.1989; 7 O $^{7} \mathrm{O}^{7}$, [3], pitfall traps 10, 21-31.VII.1989.

Intra. 15 99 , grass-dwarf shrub tundra on terrace [4], pitfall traps 10, 29.VI.-8.VII.1989; 5 ○ $^{7} \sigma^{7}, 21$ 우, [4], pitfall traps 5, 211.VII.1989; 17 Oᄋ+ [4], pitfall traps 5, 21-31.VII.1989; 4 O $^{7} 0^{7}, 15$ 우, [4], pitfall traps 10, 21-31.VII.1989; 20 O $^{7} \sigma^{7}, 40 \sigma^{7} \sigma^{7}$, [4], pitfall traps 10, 2-11.VII.1989; $40 \mathrm{O}^{0} \sigma^{7}, 70$ 우, dwarf shrubsgrass-moss-lichen tundra [5], pitfall traps 5, 2-12.VII.1989; 80 $\sigma^{7} \sigma^{7} \&$ 우, [5], pitfall traps 5, 21-31.VII.1989; 80 O $^{7} \sigma^{7}, 60$ 우, [5], pitfall traps 10, 2-11.VII.1989; 120 O $^{7} \sigma^{7} \&$ OO+, [5], pitfall traps 10, 21-31.VII.1989; 2 Oᄋ+ [5] soil sample No. 34, layer 0-0.5 cm, 18. VII.1989; $2 \mathrm{O}^{7} \mathrm{O}^{7}$, [5], soil sample No. 34, layer 0-1.5 cm, 18 . VII.1989; 1 O $^{7}$, [5], soil sample $\sigma^{7} 35$, layer $0-0.5 \mathrm{~cm}, 18$.VII.1989; 1 , [5], soil sample No. 36, layer 0-0.5 cm, 18.VII.1989; 1 , [5], soil sample No. 37, layer 0-0.5 cm, 18.VII.1989; 2 우, [5], soil sample No. 37, layer $0.5-1.5 \mathrm{~cm}, 18$.VII.1989; 1 \% [5], soil sample No. 38, layer 0-0.5 cm, 18.VII.1989; 1 \%, [5], soil sample No. 40, layer $0-1 \mathrm{~cm}, 18$.VII.1989; $25 \sigma^{7} \sigma^{7}, 40$ o+ , wet moss-cotton-grass polygonal tundra [6], pitfall traps 10, 20-31.VII.1989; 1 , swampy moss-lichen tundra in depression on watershed [7], pitfall traps 10, 22.VII.-1.VIII.1989.

RANGE. Holarctic arctic.

\section{Hilaira nivalis Holm, 1937}

MATERIAL. Zonal. $1 \%$, undershrub-moss-lichen polygonal tundra [2], pitfall traps 5, 29.VI.-8.VII.1989; $1 \bigcirc^{7}, 5$ 90 , [2], pitfall traps 10, 29.VI.-8.VII.1989; 9 O $^{7} \sigma^{7}, 17$ 우, dwarf willow-mosssedge polygonal tundra [3], pitfall traps 5, 29.VI.-8.VII.1989; 14 $0^{7} 0^{7}, 4$ ○O , [3], pitfall traps 10, 29.VI.-8.VII.1989; 1 +, [3], soil sample No. 19 , layer $0-1.5 \mathrm{~cm}, 10$.VII. 1989 .

Intra. 2 o+ , dwarf shrubs-grass-moss-lichen tundra [5], pitfall traps 5, 2-12.VII.1989; $3 \sigma^{7} \sigma^{7}, 4$ OP, [5], pitfall traps 10, 2-11. VII.1989; $43 \sigma^{7} \sigma^{7}, 27$ 웅, wet moss-cotton-grass polygonal tundra [6], pitfall traps 5, 29.VI.-8.VII.1989; $2 \sigma^{7} \sigma^{7}$, [6], pitfall traps 5, 21-31.VII.1989; 9 O $^{7}, 1$ ㅇ, [6], pitfall traps 10, 20-31.VII.1989;

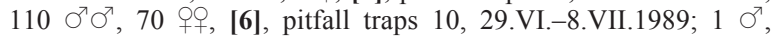


swampy moss-lichen tundra in depression on watershed [7], pitfall traps 10, 22.VII.-1.VIII.1989.

RANGE. West Siberian arctic.

\section{Hilaira proletaria (L. Koch, 1879)}

MATERIAL. Intra. $23 \sigma^{\top}, 25$ 우, wet moss-cotton-grass polygonal tundra [6], pitfall traps 5, 29.VI.-8.VII.1989; $7 \sigma^{7} \sigma^{7}$, [6], pitfall traps 5, 21-31.VII.1989; $7 \bigcirc^{7} \sigma^{7}, 4$ 우, [6], pitfall traps 10, 29.VI.-8.VII.1989; $14 \sigma^{\top} \sigma^{\top}, 1$ ㅇ, [6], pitfall traps 10, 20-31. VII.1989; $2 \bigcirc^{\top} \sigma^{7}$, swampy moss-lichen tundra in depression on watershed [7], pitfall traps 10, 22.VII.-1.VIII.1989.

RANGE. Siberian-West Nearctic.

\section{Masikia indistincta (Kulczyński, 1908)}

MATERIAL. Zonal. $1 \Im^{7}, 1$, undershrub-moss-lichen polygonal tundra [2], pitfall traps 5, 29.VI.-8.VII.1989; 1 , , [2], pitfall traps 5, 20-30.VII.1989; $11 \sigma^{\top} \sigma^{\top}, 5$ 우, [2], pitfall traps 10, 29.VI.8.VII.1989; 1 ㅇ, [2], soil sample No. 5, layer 2-4 cm, 16.VII.1989; 2 우, [2], soil sample No. 8, layer 0-2 cm, 16.VII.1989; 1 ㅇ, [2], soil sample No. 8, layer 2-4 cm, 16.VII.1989; $21 \mathrm{O}^{7} \mathrm{\sigma}^{7}, 11$ ơ, dwarf willow-moss-sedge polygonal tundra [3], pitfall traps 5, 29. VI.-8.VII.1989; $23 \sigma^{7} \sigma^{\top}, 12$ Oᄋ, [3] pitfall traps 10, 29.VI.-8.VII

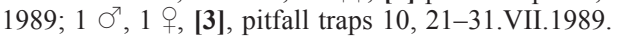

Intra. $56 \sigma^{\top} \sigma^{\top}, 4$ 우, wet moss-cotton-grass polygonal tundra [6], pitfall traps 5, 29.VI.-8.VII.1989; $2 \sigma^{7} \sigma^{7}, 1$ ㅇ, [6], pitfall traps 5, 21-31.VII.1989; $20 \sigma^{\top} \sigma^{\top}, 40$ 우, [6], pitfall traps 10, 29.VI.8.VII.1989; 71 우, [6], pitfall traps 10, 20-31.VII.1989; 1 ㅇ, [6], soil sample No. 14, layer $0-1 \mathrm{~cm}, 22$.VII.1989; 1 , , swampy mosslichen tundra in depression on watershed [7], soil sample No. 29, layer 1-4 cm, 26.VII.1989.

RANGE. Siberian-Nearctic arctic.

\section{Mecynargus tundricola Eskov, 1988}

MATERIAL. Zonal. $3 \sigma^{7} \sigma^{7}$, moss-lichen polygonal tundra [1], pitfall traps 5, 29.VI.-8.VII.1989; 1 ๙ , 1 \%, [1], pitfall traps 10, 29.VI.-8.VII.1989; 2 ठ $\sigma^{7}$, undershrub-moss-lichen polygonal tundra [2], pitfall traps 5, 29.VI.-8.VII.1989; 2 o+, [2], pitfall traps 5, 20-30.VII.1989; $12 \sigma^{7} \sigma^{7}, 6$ 우, [2], pitfall traps 10, 29.VI.-

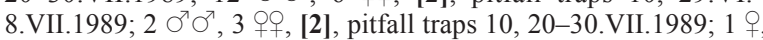
[2], soil sample No. 1, layer 0-2 cm, 16.VII.1989; 2 oㅇ, [2], soil sample No. 1, 0-2 cm, 16.VII.1989; 1 , [2], soil sample No. 3, layer 0-2 cm, 16.VII.1989; 1 , , [2], soil sample No. 2, layer 2-4 cm, 16.VII.1989; $4 \sigma^{7} \sigma^{7}$, dwarf willow-moss-sedge polygonal tundra [3], pitfall traps 5, 29.VI.-8.VII.1989; 2 \%+, [3], pitfall traps 5, 21-31.VII.1989; 5 Oᄋ, [3], pitfall traps 10, 21-31.VII.1989.

Intra. $1 \sigma^{7}$, grass-dwarf shrub tundra on terrace [4], pitfall traps 10, 2-11.VII.1989; 2 ऽ $\sigma^{7}$, [4], pitfall traps 10, 29.VI.8.VII.1989; $2 \Im^{7} \sigma^{7}$, dwarf shrubs-grass-moss-lichen tundra [5] pitfall traps 10, 2-11.VII.1989; 2 ○ $\sigma^{\top}$, [5], pitfall traps 5, 2131.VII.1989; 1 , wet moss-cotton-grass polygonal tundra [6], pitfall traps 5, 29.VI.-8.VII.1989; $29 \sigma^{7} \sigma^{7}, 41$ O0, [6], pitfall traps 10 20-31.VII.1989; 1 , [6], soil sample No. 14, layer $1-4 \mathrm{~cm}, 22$.VII. 1989; $1 \sigma^{7}$, swampy moss-lichen tundra in depression on watershed [7], pitfall traps 10, 22.VII.-1.VIII.1989; $1 \sigma^{7}$, [7], soil sample No. 27, layer $1-4 \mathrm{~cm}, 25$.VII.1989.

RANGE. Siberian arctic.

\section{Pelecopsis parallela (Wider, 1834)}

MATERIAL. Zonal. $1+$, dwarf willow-moss-sedge polygonal tundra [3], pitfall traps 10, 21-31.VII.1989.

RANGE. Palaearctic polyzonal.

\section{Semljicola arcticus (Eskov, 1989)}

MATERIAL. Zonal. $3 \bigcirc^{7} \sigma^{7}$, moss-lichen polygonal tundra [1], pitfall traps 5, 29.VI.-8.VII.1989; 1 ๙ , 1 \%, [1], pitfall traps 10,
29.VI.-8.VII.1989; $4 \sigma^{7} \sigma^{7}, 8$ 80 , undershrub-moss-lichen polygo-

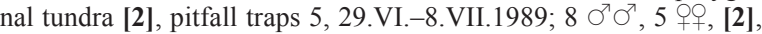
pitfall traps 5, 20-30.VII.1989; $15 \sigma^{\top} \sigma^{\top}, 5$ 우, [2], pitfall traps 10, 29.VI.-8.VII.1989; 6 ○ $^{7}, 6$ 우, [2], pitfall traps 10, 2030.VII.1989; 1 क, [2], soil sample No. 2, layer 2-4 cm, 16.VII.1989; $5 \bigcirc^{\top} \sigma^{\top}, 2$ 우, dwarf willow-moss-sedge polygonal tundra [3], pitfall traps 5, 29.VI.-8.VII.1989; $3 \sigma^{7} \sigma^{7}, 3$ oᄋ, [3], pitfall traps 5, 21-31.VII.1989; $7 \sigma^{\top} \sigma^{\top}, 4$ क⿱, [3], pitfall traps 10, 29.VI.-8.

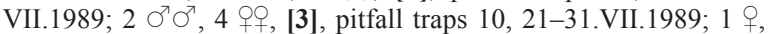
[3], soil sample No. 17, layer 0-1 cm, 10.VII.1989.

Intra. $1 \sigma^{T}, 3$ 우, dwarf shrubs-grass-moss-lichen tundra [5], pitfall traps 5, 21-31.VII.1989; $3 \bigcirc^{7} \sigma^{7}, 2$ 우, [5], pitfall traps 10, 2-11.VII.1989; $3 \sigma^{\top} \sigma^{\top}$, wet moss-cotton-grass polygonal tundra [6], pitfall traps 5, 29.VI.-8.VII.1989; $5 \sigma^{7} \sigma^{7}$, [6], pitfall traps 10, 29.VI.-8.VII.1989.

RANGE. Siberian arctic.

\section{Results and Discussion}

\section{Interesting records}

Among findings on the island, only one can be perhaps considered as interesting: Agyneta decora, a rare polyzonal element of the arctic island fauna, which is known, besides the Sibiryakova Island, only from Dolgiy Island [Marusik et al., 2016], see details above in the List of spiders.

The Sibiryakova Island is situated in the vicinities of the Yenisei River mouth, and it would be logical to suggest that the river flow with driftwoods (see above) or with trash brings towards the island the elements of more southern, hypoarctic and/or boreal faunas. Regretfully, we were unable to reveal any of such species. Probably, they do exist but live in marsh environments, which have not been covered by our investigations, or they simply cannot survive in the very pessimal conditions on the island, even having been introduced.

\section{Fauna and population composition}

In total, 13 spider species have been collected on the island. All of them belong to the family Linyphiidae and represented by two zonal/latitudinal fractions: arctic fraction (11 species) and polyzonal fraction (two species), see details in Tanasevitch \& Khruleva [2017]. All species from the arctic fraction are purely arctic elements and in classification/typification of Chernov [1978], a part of them can be characterized as euarctic: Arcterigone pilifrons, Diplocephalus barbiger, Erigone psychrophila, Halorates spetsbergensis, Hilaira proletaria, Semljicola arcticus; or as hemiarctic: Erigone arctica palaearctica, Gibothorax tchernovi, Hilaira nivalis, Masikia indistincta and Mecynargus tundricola. Two species, Agyneta decora and Pelecopsis parallela are polyzonal.

The absence of wolf-spiders, Lycosidae, large and active predators, was not surprising. Lycosidae are absent also on nearby (again in the subzone of arctic tundras) Shokalsky Island [Nekhaeva, 2018]. They were not found also on Vaygach Island situated much southerner, at the border between Kara and Pechora seas [Tanasevitch, 2017b]. Lycosidae are known only from 
Table 1 . Spider records by pitfall traps.

Таблица 1. Данные учёта пауков почвенными ловушками.

\begin{tabular}{|c|c|c|c|c|c|c|}
\hline \multirow{5}{*}{ Species } & \multicolumn{6}{|c|}{ Types of plant communities } \\
\hline & \multicolumn{3}{|c|}{ Zonal communities } & \multicolumn{3}{|c|}{ Intrazonal communities } \\
\hline & \multicolumn{6}{|c|}{ Plots in tundra } \\
\hline & No. 1 & No. 2 & No. 3 & No. 4 & No. 5 & No. 6 \\
\hline & \multicolumn{6}{|c|}{ Number of collected specimens } \\
\hline Agyneta decora & & & & 2 & & \\
\hline Arcterigone pilifrons & & & 35 & & & 7 \\
\hline Diplocephalus barbiger & & 1 & & 9 & 15 & 126 \\
\hline Erigone a. palaearctica & & & 20 & 59 & & \\
\hline E. psychrophila & 2 & 14 & 129 & 39 & 26 & 143 \\
\hline Gibothorax tchernovi & & & & & & 97 \\
\hline Halorates spetsbergensis & 279 & 40 & 24 & 137 & 450 & 65 \\
\hline Hilaira nivalis & & 7 & 44 & & 9 & 262 \\
\hline H. proletaria & & & & & & 81 \\
\hline Masikia indistincta & & 19 & 69 & & & 194 \\
\hline Mecynargus tundricola & 5 & 27 & 11 & 3 & 4 & 71 \\
\hline Pelecopsis parallela & & & 1 & & & \\
\hline Semljicola arcticus & 5 & 57 & 30 & & 9 & 8 \\
\hline Total specimens & 291 & 165 & 363 & 249 & 513 & 1054 \\
\hline Total species & 4 & 7 & 9 & 6 & 6 & 10 \\
\hline
\end{tabular}

Dolgy Island [Marusik et al., 2016] situated even southward - in the subzone of southern tundras and having recent connection with closely situated continent. Wolfspiders on Dolgy Island are represented by five species [Marusik et al., 2016], of which only one species is arctic, and the other belong to the boreal fraction. The absence of Lycosidae on arctic islands can be explained by severe conditions of arctic tundras which do not allow them to survive until the adult state. It can be also suggested the presence of a strong pressure of birds which actively eat out these large spiders, especially during the nestling breeding. It can be noted also that the fauna of Ragozinka (mainland Taimyr), situated at about the same latitude in $40-50 \mathrm{~km}$ eastward of the Sibiryakova, was characterized by presence of at least one species of Lycosidae - the arctic species Alopecosa mutabilis (Kulczyński, 1908) [Eskov, 1985]. However, it cannot be excluded that the borders of tundra subzones in West Taimyr, as compared to that, are somewhat southward shifted, and Ragozinka, in the opinion of Matveyeva \& Zanokha [1997], is situated near the border between typical and arctic tundras. It cannot be excluded that the absence of Lycosidae representatives in the fauna is a certain marker of arctic tundras.

Therefore, in the composition of zoogeographic elements, the fauna of the island can be characterized as
Siberian and mostly composed of Siberian and Siberian-Nearctic ranges. In the composition of zonal fractions of the island fauna, it is arctic and is represented by purely arctic elements (11 species) at a small inclusion of polyzonal species (2). Hypoarctic and boreal elements are absent.

Among purely arctic elements, three species, Erigone psychrophila, Halorates spetsbergensis and Mecynargus tundricola, are dominants widely explore all the biotopes, both zonal (most pessimal) and intrazonal, though being most abundant in the latter ones (see Table 1). Arctic Semljicola arcticus was not found in only one (intrazonal) biotope, though, on the contrary, it is most abundant in the zonal communities. Polyzonal Agyneta decora and Pelecopsis parallela, have been found only as singletons in intrazonal and zonal communities, respectively.

The most pessimal community — the zonal mosslichen polygonal tundra - as compared to other areas, is characterized by the least number of species found (4) and their least dynamic density (see Tables 1 \& 2). All these typical arctic faunistic elements were found in the zonal polygonal tundra only as single specimens, except the only case, Halorates spetsbergensis, which probably realized here its reproductive abilities at the situation of lack of the competition, despite the pessimal living conditions. Two other zonal communities, 
Table 2. Spider records by pitfall traps: dynamic density/activity of spiders (per 100 trap-days). Таблица 2. Данные учёта почвенными ловушками: динамическая плотность/активность пауков (на 100 ловушко-суток).

\begin{tabular}{|c|c|c|c|c|c|c|}
\hline & \multicolumn{6}{|c|}{ Types of plant communities } \\
\hline & \multicolumn{3}{|c|}{ Zonal communities } & \multicolumn{3}{|c|}{ Intrazonal communities } \\
\hline & \multicolumn{6}{|c|}{ Plots in tundra } \\
\hline & No. 1 & No. 2 & No. 3 & No. 4 & No. 5 & No. 6 \\
\hline Traps positioning period & $\begin{array}{l}\text { 29.VI- } \\
\text { 8.VII }\end{array}$ & $\begin{array}{l}\text { 29.VI- } \\
\text { 8.VII }\end{array}$ & $\begin{array}{l}\text { 29.VI- } \\
\text { 8.VII }\end{array}$ & $\begin{array}{c}2- \\
12 . \mathrm{VII}\end{array}$ & $\begin{array}{c}2- \\
12 . \mathrm{VII}\end{array}$ & $\begin{array}{l}29 . \mathrm{VI}- \\
8 . \mathrm{VII}\end{array}$ \\
\hline $\begin{array}{l}\text { Number of collected spiders per } \\
100 \text { trap-days }\end{array}$ & 97 & 101 & 173 & 198 & 258 & 458 \\
\hline Traps positioning period & $\begin{array}{c}20- \\
30 . \text { VII }\end{array}$ & $\begin{array}{c}20- \\
30 . \text { VII }\end{array}$ & $\begin{array}{c}21- \\
31 . \text { VII }\end{array}$ & $\begin{array}{c}21- \\
31 . \mathrm{VII}\end{array}$ & $\begin{array}{c}21- \\
31 . \text { VII }\end{array}$ & $\begin{array}{c}21- \\
31 . \mathrm{VII}\end{array}$ \\
\hline $\begin{array}{l}\text { Number of collected spiders per } \\
100 \text { trap-days }\end{array}$ & 154 & 29 & 107 & 80 & 184 & 312 \\
\hline
\end{tabular}

Table 3. Spider records by soil samples (spms/sq. m). Таблица 3. Данные учёта пауков почвенными пробами (в экз./кв. м).

\begin{tabular}{|c|c|c|c|c|c|c|}
\hline \multirow{5}{*}{ Species } & \multicolumn{6}{|c|}{ Types of plant communities } \\
\hline & \multicolumn{3}{|c|}{ Zonal communities } & \multicolumn{3}{|c|}{ Intrazonal communities } \\
\hline & \multicolumn{6}{|c|}{ Plots in tundra } \\
\hline & No. 1 & No. 2 & No. 3 & No. 4 & No. 5 & No. 6 \\
\hline & \multicolumn{6}{|c|}{ Number of collected specimens } \\
\hline \multicolumn{7}{|l|}{ Agyneta decora } \\
\hline Arcterigone pilifrons & & & 6 & & & \\
\hline \multicolumn{7}{|l|}{ Diplocephalus barbiger } \\
\hline \multicolumn{7}{|l|}{ Erigone a. palaearctica } \\
\hline \multicolumn{7}{|l|}{ E. psychrophila } \\
\hline \multicolumn{7}{|l|}{ Gibothorax tchernovi } \\
\hline Halorates spetsbergensis & & & & & 22 & \\
\hline Hilaira nivalis & & & 2 & & & \\
\hline \multicolumn{7}{|l|}{ H. proletaria } \\
\hline Masikia indistincta & & 8 & & & & 2 \\
\hline Mecynargus tundricola & & 10 & & & & 2 \\
\hline \multicolumn{7}{|l|}{ Pelecopsis parallela } \\
\hline Semljicola arcticus & & 2 & 2 & & & \\
\hline Aranei juv. & & 26 & 20 & & 12 & 32 \\
\hline Spider quantity per 1 sq. m. & $*$ & 46 & 30 & $*$ & 34 & 36 \\
\hline
\end{tabular}

* due to a strong impounding of the plots, the soil samples were not taken in due course.

undershrub-moss-lichen- and dwarf willow-moss-sedge polygonal tundras, were much richer in species ( 7 and 9 , respectively) and showed a considerably higher catching efficiency of the pitfall traps, which probably is connected with their higher humidity.

Intrazonal vegetable communities are generally more humid and are characterized by less pessimal living conditions as compared to zonal ones. This probably explains their richer faunal composition (12 vs. 10) and much more abundant population of spiders: the catching efficiency of the pitfall traps in intrazonal communities is 2.2 times higher than in the zonal ones (see Tables $1 \& 2$ ). The richest in the number of species (10) and the population density is the moistened moss-cotton grass polygonal tundra (plot No. 6). Just in this area many species reach their maximal abun- 
Table 4. Spider abundance (spms/sq. m) in soil samples and their share in the total population of macrofauna. Таблица 4. Численность пауков (экз./кв. м) в почвенных пробах и их доля в общем населении мезофауны.

\begin{tabular}{|l|c|c|c|c|c|}
\hline \multirow{2}{*}{} & \multicolumn{5}{|c|}{ Types of plant communities } \\
\cline { 2 - 6 } & \multicolumn{2}{|c|}{ Zonal communities } & \multicolumn{3}{c|}{ Intrazonal communities } \\
\cline { 2 - 6 } & \multicolumn{5}{|c|}{ Plots in tundra } \\
\cline { 2 - 6 } & No. 2 & No. 3 & No. 5 & No. 6 & No. 7 \\
\hline Aranei (spms/sq. m) & 46 & 30 & 34 & 36 & 10 \\
\hline $\begin{array}{l}\text { Share of spiders in the total population } \\
\text { of macrofauna }\end{array}$ & $51 \%$ & $18 \%$ & $63 \%$ & $27 \%$ & $14 \%$ \\
\hline $\begin{array}{l}\text { Total abundance of macrofauna } \\
\text { (spms/sq. m) }\end{array}$ & 92 & 166 & 52 & 136 & 74 \\
\hline
\end{tabular}

dance (as expressed in dynamic density): Diplocephalus barbiger, Erigone psychrophila, Hilaira nivalis, H. proletaria, and Gibothorax tchernovi. The two latter species live only there and are rather abundant.

This study confirms the conclusions of many authors (see Tanasevitch, Rybalov, 2015; Tanasevitch, Nekhaeva, 2016; Tanasevitch, Khruleva, 2017; Khruleva et al., 2018, etc.) that humid intrazonal biotopes possess a considerably richer spider fauna, their population is more abundant and represented by a wider array of latitudinal fractions, as compared to other types of vegetable communities of arctic tundras.

\section{Spiders in the soil macrofauna structure}

Accounts by the pitfall traps and soil samples have shown that spiders are the main group among population of the soil macrofauna of local arctic tundras: they were dominant in almost all biotopes studied. Thus, the proportion of spiders comprised $27-63 \%$ in most plots, and only the plot No. 7 was different in the marginal participation of spiders in the total population $(14 \%)$, less of that of most other soil invertebrates, which can be explained by marked impoundment of this plot in 1989 (see Table 4). As compared to data from pitfall traps, the taxonomic composition of spiders in the soil samples was much poorer: the samples revealed only seven species of 13 (see Table 3). Significantly, all these species from the trap data were dominant in the same biotopes, that is the soil samples found only the most abundant species of a certain biocoenosis (see Table 1).

Spiders are the dominant group in the soil macrofauna in local arctic tundras: during the season they mainly account for 30 to $60 \%$ of the total population of soil macrofauna. The proportion of spiders changes in dependence on the vegetation period terms: in the beginning (late June to early July) it comprised 60-91\%, in the second half of summer - 10-49\%. Spiders are the first group of large soil invertebrates becoming active just after the snow melting. By the late July, almost all biocoenoses contain either just appeared or becoming active other invertebrate groups, which were before in a diapause or in a latent condition (inactive juveniles).

Acknowledgements. This study was supported by the Presidium of the Russian Academy of Sciences, Program No.41 "Biodiversity of natuarl systems and biological recources of Russia".

\section{References}

Bulavintsev V.I., Babenko A.B. 1983. [Soil-dwelling invertebrates in the eastern sector of Franz Josef land] // Zool. Zhurn. Vol.62. No.7. P.1114-1116 [in Russian].

Chernov Yu.I. 1978. [The structure of the animal population of the Subarctic]. Moscow: Nauka Publ. 165 pp. [In Russian]

Dupérré N. 2013. Taxonomic revision of the spider genera Agyneta and Tennesseelum (Araneae, Linyphiidae) of North America north of Mexico with a study of the embolic division within Micronetinae sensu Saaristo \& Tanasevitch 1996 // Zootaxa. Vol. 3674. P.1-189.

Eskov K.Yu. 1985. [Spiders of the tundra zone of the USSR] // Ovcharenko V.I. (ed.). Fauna i ekologiya paukov SSSR. Trudy Zool. Inst. Akad. nauk SSSR, Leningrad. Vol.139. P.121-128 [in Russian].

Holm Å. 1970. Notes on spiders collected by the "Vega" Expedition 1878-1880. Entomologica Scandinavica. Vol.1. No.3. P.188-208.

Khruleva O.A. 1987. [Invertebrates] // Flora i fauna zapovednikov SSSR: Fauna zapovednika "Ostrov Wrangelya". Moscow. P.636 [in Russian].

Khruleva O.A., Tanasevitch A.V., Dondua A.G., Vartanyan N.I. 2018. Spiders (Aranei) of the Belyaka Spit, Chukotka: peculiarities of the fauna and biotopic distribution // Arthropoda Selecta. Vol.27. No.4. P.373-380.

Kuvaev V.B., Koshevnikova A.D., Gudoshnikov S.V., Shurbenko M.P., Nezdoyminogo E.L. 1994. [Vegetation cover of the Sibiryakova Island] // Opyt kompleksnogo floristicheskogo i geobotanicheskogo issledovaniya. Moscow. 138 pp. [In Russian]

Marusik Yu.M., Koponen S., Makarova O.L. 2016. A survey of spiders (Araneae) collected on the arctic island Dolgiy $\left(69^{\circ} 12^{\prime} \mathrm{N}\right)$, Barents Sea // Arachnology. Vol.17. No.1. P.1024.

Marusik Yu.M., Eskov K.Yu., Koponen S., Vinokurov N.N. 1993. A check-list of the spiders (Aranei) of Yakutia, Siberia // Arthropoda Selecta. Vol.2. No.2. P.63-79.

Matveyeva N.V. 1998. [Zonation in plant cover of the Arctic] // Trudy Botanicheskogo instituta RAN. Vol.21. St.-Petersburg. 220 p. [In Russian]

Matveyeva N.V., Zanokha L.L. 1984. [Some notes on the flora and vegetation of the Sibiryakov Island] // Botanic Journal. Vol.70. No.5. P.616-624 [in Russian]. 
Matveyeva N.V., Zanokha L.L. 1997. [Vascular flora of the northwestern part of the Taimyr Peninsula]// Botanic Journal. Vol.82. No.12. P.11-19 [in Russian].

Nekhaeva A.A. 2018. Spiders (Arachnida, Aranei) of the High Arctic Shokalsky Island $\left(73^{\circ} \mathrm{N}\right)$, the Kara Sea, Russia // Arthropoda Selecta. Vol.27. No.4. P.367-372.

Nekhaeva A.A., Nekhaev I.O. 2011. On the spider fauna of Bolshoy Aynov Island (Barents Sea) (Arachnida: Aranei) // Arthropoda Selecta. Vol.20. No.4. P.319-322.

Osipov D.V. 2003. [Structure of the spider (Aranei) fauna in southern tundra of western Taimyr] // Zool. zhurn. Vol.82. No.10. P.1266-1270 [in Russian].

Tanasevitch A.V. 2017a. New records of spiders (Aranei) from the Russian Arctic // Arthropoda Selecta. Vol.26. No.1. P.77-82.

Tanasevitch A.V. 2017b. Spiders (Aranei) of the Novaya Zemlya Archipelago and the Vaygach Island, Russia // Arthropoda Selecta. Vol.26. No.2. P.145-153.
Tanasevitch A.V. 2018. New data on spiders (Aranei) from the Novaya Zemlya Archipelago and Siberian Arctic, Russia // Arthropoda Selecta. Vol.21. No.1. P.69-71.

Tanasevitch A.V., Khruleva O.A. 2017. Spiders (Aranei) of the typical tundra subzone of the Yugorsky Peninsula, Russia // Arthropoda Selecta. Vol.26. No.4. P.341-368.

Tanasevitch A.V., Koponen S. 2007. Spiders (Aranei) of the southern tundra in the Russian Plain // Arthropoda Selecta. Vol.15 (for 2006). No.4. P.295-345.

Tanasevitch A.V., Nekhaeva A.A. 2016. Spiders (Aranei) of the Kharaulakh Mountains and the Lena River lower reaches, northern Yakutia, Russia// Arthropoda Selecta. Vol.25. No.3. P.307326.

Tanasevitch A.V., Rybalov L.B. 2015. Fauna and distribution of spiders (Aranei) of the arctic tundra in northern Yamal Peninsula, Russia // Arthropoda Selecta. Vol.24. No.2. P.215-230.

Responsible editor K.G. Mikhailov 